\title{
A generalizable NLP framework for fast development of pattern-based biomedical relation extraction systems
}

Yifan Peng ${ }^{1 *}$, Manabu Torii ${ }^{1,2}$, Cathy $\mathrm{H} \mathrm{Wu}^{1,2}$ and K Vijay-Shanker ${ }^{1}$

\begin{abstract}
Background: Text mining is increasingly used in the biomedical domain because of its ability to automatically gather information from large amount of scientific articles. One important task in biomedical text mining is relation extraction, which aims to identify designated relations among biological entities reported in literature. A relation extraction system achieving high performance is expensive to develop because of the substantial time and effort required for its design and implementation. Here, we report a novel framework to facilitate the development of a pattern-based biomedical relation extraction system. It has several unique design features: (1) leveraging syntactic variations possible in a language and automatically generating extraction patterns in a systematic manner, (2) applying sentence simplification to improve the coverage of extraction patterns, and (3) identifying referential relations between a syntactic argument of a predicate and the actual target expected in the relation extraction task.

Results: A relation extraction system derived using the proposed framework achieved overall F-scores of $72.66 \%$ for the Simple events and 55.57\% for the Binding events on the BioNLP-ST 2011 GE test set, comparing favorably with the top performing systems that participated in the BioNLP-ST 2011 GE task. We obtained similar results on the BioNLP-ST $2013 \mathrm{GE}$ test set (80.07\% and 60.58\%, respectively). We conducted additional experiments on the training and development sets to provide a more detailed analysis of the system and its individual modules. This analysis indicates that without increasing the number of patterns, simplification and referential relation linking play a key role in the effective extraction of biomedical relations.

Conclusions: In this paper, we present a novel framework for fast development of relation extraction systems. The framework requires only a list of triggers as input, and does not need information from an annotated corpus. Thus, we reduce the involvement of domain experts, who would otherwise have to provide manual annotations and help with the design of hand crafted patterns. We demonstrate how our framework is used to develop a system which achieves state-of-the-art performance on a public benchmark corpus.
\end{abstract}

\section{Background}

Due to the continued growth of biomedical publications, it has become very difficult for scientists to keep up with the new findings reported in the literature. As a consequence, we have observed an increase in the effort spent on automatically extracting information from research literature and developing biomedical text mining tools.

\footnotetext{
*Correspondence: yfpeng@udel.edu

${ }^{1}$ Department of Computer and Information Sciences, University of Delaware, 18 Amstel Ave, Newark, DE 19716, USA

Full list of author information is available at the end of the article
}

This paper aims to address the relation extraction task, which identifies selected types of relationships among entities (e.g., proteins) reported in text.

Approaches to the relation extraction task can be categorized into two major classes: (1) machine learningbased approaches and (2) pattern-based approaches. Machine learning-based approaches are data-driven and can derive models from a set of annotated data [1-7]. The use of machine learning methods can be quite effective, but the performance of resulting systems depends on the quality and the amount of annotated data. For example, large annotated corpora become available for the protein-protein interaction relation task, reflecting a 
general community-wide interest [8]. But this situation does not always hold for relations of different scientific interest, because preparing annotated corpora is generally time consuming and expensive and it also requires domain expertise and significant effort to ensure accuracy and consistency. In contrast, pattern-based approaches do not require annotated data to train a system. However, they do require domain experts to be closely involved in the design and implementation of the system to capture the patterns used for extracting the necessary information. Some systems rely on extraction patterns defined at the surface textual level or based on outputs from a shallow parser [9-12]. Others use deep parsers with hand-crafted patterns [13-17]. As found in OpenDMAP [18], a semantic grammar may be utilized with text literals, syntactic constituents, semantic types of entities, and hyponomy. In all cases, rigid extraction patterns are manually encoded in the systems. Owing to rigid patterns, pattern-based approaches usually achieve a high precision but are often cited for low recall. While it is feasible to manually identify and implement high quality patterns to achieve a good precision, it is often impractical to exhaustively encode all the patterns necessary for a high recall in this manner.

Our work enables the fast development of pattern-based systems, while mitigating some of these concerns. We aim to reduce the involvement of domain experts and their manual annotation, and to attain high precision and recall.

Our approach starts by identifying a list of trigger words for the target relation (e.g., "associate" for the binding relation) and their corresponding Trigger specifications (e.g., the number and type of arguments expected for each trigger). Given this information, we make use of linguistic principles to derive variations of lexico-syntactic patterns in a systematic manner. These patterns are matched with the input text in order to extract target relations.

To improve the applicability of the generated patterns, we incorporate two additional design features. The first is the use of text simplification. This allows us to design a small set of lexico-syntactic patterns to match simple sentence constructs, rather than try to account for all complex syntactic constructs by generating an exhaustively large amount of patterns. Second, the framework exploits referential relations. With this method, two phrases referring to the same entity (e.g., coreference relation) or in a particular relation (e.g., meronymy relation, also known as part-of relation) are detected in text, and links are established between them. These links can be used when seeking the most appropriate phrase referring to the target entity and, hence, allow for extraction of target entities beyond lexico-syntactic patterns.

The proposed approach is based on the property of the language, rather than task-specific knowledge. Therefore, it is generalizable for different trigger words and potentially applicable to many different types of information targeted in biomedical relation extraction tasks.

We acknowledge several studies underlying our proposed framework. The automated pattern generation employed in this study shares the fundamental assumptions of certain linguistic theories, such as Lexicalized Tree Adjoining Grammar (LTAG) [19], Head-Driven Phrase Structure Grammar [20], and Lexical Functional Grammar [21]. In particular, we believe that the concept underlying our method is similar to that of LTAG. The paradigm of inferring patterns exploited in our method shares the ideas with [22-30], but we focus on a specific set of patterns pertaining to the expression of biomedical relations.

Simplifying a sentence as a prerequisite for biomedical information extraction was studied in the past $[9,11,31-34]$. The use of meronymy and its opposite holonymy, among other relationships found in the biomedical ontology, was discussed in [35]. Some of these relations were later considered in biomedical information extraction systems in order to improve their performance [36-38]. These relations and paradigms are in conjunction with our own two additional referential relationships: coreference and hyponymy. We integrate them in our framework and examine their utility for biomedical relation extraction.

To evaluate the framework, we test it by producing an extraction system for six relations that were part of the BioNLP-ST 2011 and 2013 GE tasks. We show that by just taking the specification of trigger words (root word only), we produce a relation extraction system with results that compare favorably with state-of-the-art results on this corpus. We further show that we can achieve good precision and recall with the patterns generated from the trigger, and that simplification and referential relation linking can increase the recall without compromising the precision.

\section{Methods}

\section{A. Architecture overview}

The architecture of our framework has several components (Figure 1), as summarized below and detailed in sections B-F. The framework consists of four system modules (Pattern generation, Pattern matching, Sentence simplification, and Referential relation linking) and two external modules (Parsing and Entity typing).

It also requires Trigger specifications and associated Pattern templates to locate the relations of interest. An example trigger specification is shown below:

(1) Trigger: phosphorylate 1 $\langle$ type $\rangle=$ phosphorylation $\quad 2$ $\langle$ frame $\rangle=$ Frame: $N P_{0} / N P_{1} \quad 3$ $\left\langle N P_{0}\right.$ type $\rangle=$ gene or gene product 4 $\left\langle N P_{1}\right.$ type $\rangle=$ gene, gene product or protein part $\quad 5$ $\left\langle N P_{0}\right.$ role $\rangle \leftarrow$ agent

$\left\langle N P_{1}\right.$ role $\rangle \leftarrow$ theme 


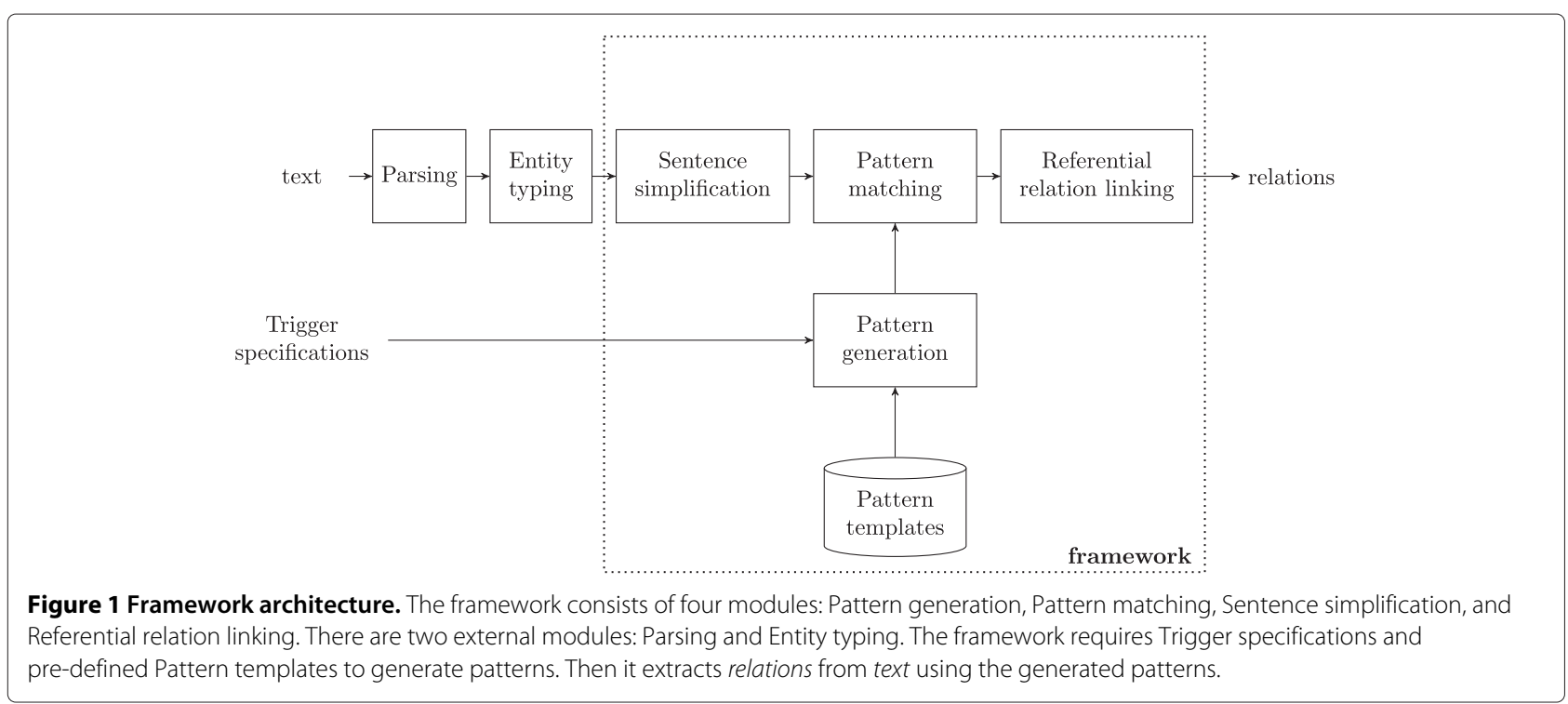

In the above example, Line 1 shows the trigger word, "phosphorylate" in this case. Line 2 indicates that it is the trigger for the relation "phosphorylation". Line 3 specifies that the trigger syntactically chooses two noun phrases, designated as $\mathrm{NP}_{0}$ and $\mathrm{NP}_{1}$. Lines $4-5$ add selectional restrictions, by requiring $\mathrm{NP}_{0}$ to be a gene or gene product (GGP) and $\mathrm{NP}_{1}$ to be either a GGP or a protein part. Lines 6-7 show that if $\mathrm{NP}_{0}$ and $\mathrm{NP}_{1}$ can be extracted, and if both $\mathrm{NP}_{0}$ and $\mathrm{NP}_{1}$ meet the above constraints, then the framework will assign their semantic roles of agent and theme, respectively.

Now consider the following example sentence:

(2) The c-Jun amino-terminal kinase phosphorylates NFAT4.

From (2), we will extract "the c-Jun amino-terminal kinase" as the agent and "NFAT4" as the theme of the phosphorylation relation. This extraction is the result of matching the text fragment with a pattern that is partly derived from the trigger specification. This pattern should not only capture the general syntactic form of a clause involving a transitive verb in an active voice, but also capture the selection restrictions imposed by the word "phosphorylates" and the arguments. Thus, this pattern contains information described in two places: (1) lexical trigger that specifies the arguments, the selection restrictions on the argument, and the role they play, and (2) the syntactic constraints corresponding to different constructs (in this example, the active clause). We call the former "trigger specification", and the latter "pattern templates". Actual lexico-syntactic patterns are obtained by merging the trigger specifications and pattern templates. As we shall see later (section B), the combination of these two is mediated by the frame that is mentioned in the trigger specification.

We now briefly discuss four modules of the system framework: Pattern generation, Pattern matching, Sentence simplification, and Referential relation linking (Figure 1).

The Pattern generation (section $C$ ) module uses trigger specifications and predefined pattern templates to derive lexico-syntactic patterns for each trigger word. The Pattern matching (section D) module then matches fragments of text with lexico-syntactic patterns, and extracts the textual expressions in the trigger and argument positions. In order to more effectively match with the patterns, the Sentence simplification (section E) module is used to preprocess the input text. It aims to ensure that the lexico-syntactic patterns generated in the previous step are able to be matched even in complex sentences. Finally, the Referential relation linking (section F) module links arguments identified by the pattern matching module with the target entities they refer to, where applicable. This step enables the system to find relations between more appropriate entities than the ones referred by textual expressions in the argument position.

In addition to the above four system modules, there are two external modules. One is the Parsing module, which is used by the pattern matching step. The other is the Entity typing module, which assigns semantic types or categories to noun phrases. Both are found to be useful to enhance the precision of the relation extraction task $[12,18,39]$.

\section{B. Trigger specification}

Trigger specifications are used to locate triggers and arguments in text for target relations. To make it easier to 
specify triggers, we ask users to provide the trigger's root, which is the primary lexical unit of a word. From the root morpheme, we can derive other forms of triggers using our previous work [40]. For example, from "phosphorylate" we derive "phosphorylates", "phosphorylated", "phosphorylation", etc. In general, we generate different possible forms of triggers and confirm whether they are used in the literature. In a few cases, we ask the users for this confirmation. This generation is based on well-known English inflection rules, and this can be used to match to the appropriate trigger template.

Next, we show two example trigger specifications for the same root morpheme, "express", but with different semantic types for the argument, gene and RNA.

$$
\begin{aligned}
& \text { Trigger: express.01 } \\
& \langle\text { relation }\rangle=\text { Gene_expression } \\
& \langle\text { frame }\rangle=\text { Frame: } N P_{0} / N P_{1} \\
& \left\langle N P_{1} \text { type }\right\rangle=\text { gene } \\
& \left\langle N P_{1} \text { role }\right\rangle \leftarrow \text { theme }
\end{aligned}
$$

(4) Trigger: express.02

$$
\begin{aligned}
& \langle\text { relation }\rangle=\text { Transcription } \\
& \langle\text { frame }\rangle=\text { Frame: } N P_{0} / N P_{1} \\
& \left\langle N P_{1} \text { type }\right\rangle=R N A \\
& \left\langle N P_{1} \text { role }\right\rangle \leftarrow \text { theme }
\end{aligned}
$$

Although these two specifications share the same trigger word, they represent different types of relations: gene expression and transcription. The gene expression relation requires its theme $\left(\mathrm{NP}_{1}\right)$ to be a gene, whereas the transcription relation requires its theme $\left(\mathrm{NP}_{0}\right)$ to be an RNA. These examples show that argument types in the trigger specification are essential to the framework to achieve a high precision, because they emphasize the selection restrictions on arguments.

\section{Pattern generation}

Provided with a trigger specification, we use the "frame" to associate a trigger with a set of pattern templates to derive lexico-syntactic patterns. In the following subsections, we will define frames and pattern templates, and then discuss how they can be combined to generate lexico-syntactic patterns.

\section{C.1 Frames}

A frame is a set of pattern templates sharing the same syntactic nature of the constituents that are likely to be associated with the trigger. It specifies the arguments of the trigger. We found that the most frequent frame in biomedical documents is:

(5) Frame: $N P_{0} / N P_{1}$
We distinguish $\mathrm{NP}_{0}$ and $\mathrm{NP}_{1}$, because semantically they play different roles and have different types in the trigger specification, and syntactically they represent different grammatical constituents. The above frame may be realized by the standard active form " $\mathrm{NP}_{0} \mathrm{~V} \mathrm{NP}_{1}$ ", where $\mathrm{V}$ is a verb, and $\mathrm{NP}_{0}$ and $\mathrm{NP}_{1}$ appear at the left and right of the verb, respectively.

Relations can be semantically "directional" or "nondirectional". For example, phosphorylation is a directional relation, but binding is non-directional. This is because "A binds B" and "B binds A" may be used to specify the same relation between "A" and " $\mathrm{B}$ ", but "A phosphorylates $\mathrm{B}$ " and "B phosphorylates $\mathrm{A}$ " represent two different relations. If a relation is directional (or nondirectional), we would expect that all triggers for that relation have the property as well. In our framework, we use an additional binary constraint " direction〉 = directional/non-directional" in the trigger specification to distinguish non-directional relations from the other, because currently it is the only place where users interact with the framework. To generate appropriate patterns, this directionality constraint in the trigger specification will cause an appropriate defined frame to be chosen: the non-directional frame differs from the directional one by allowing for the swapping of the agent and the theme.

\section{C.2 Pattern templates}

A pattern template is specified by a sequence of words or phrases $\beta_{1}, \ldots, \beta_{n}$, followed by a set of constraints. Each constraint assigns a value for one of the $\beta_{i}$ features.

To reduce the number of pattern templates, we limit pattern templates to capture one argument at a time. So the pattern templates will capture pairs $<$ trigger, $\mathrm{NP}_{\mathrm{i}}>$. After templates are instantiated and arguments are extracted, we combine pairs if they have the same trigger. Thus we can extract relations with multiple arguments. We believe that considering one argument at a time is more flexible and manageable, because such pairs can be applied independently, while constraints on combinations can cover many different relations.

We further categorize pattern templates into two groups: one with explicit argument, and one with null argument. We will discuss pattern templates for argument realization in the next section, and then introduce methods to generate lexico-syntactic patterns. Lastly, we will discuss pattern templates with null argument.

\section{C.3 Pattern templates for argument realization}

Argument realization, which is at the heart of the area of linguistics, is the study of the possible syntactic expressions of the arguments of a verb [41]. In this study, we extend argument realization to nominal and adjectival triggers derived from verbs as well. 
Verbal triggers Below are examples of pattern templates for verbal predicate $V_{\text {tr }}$ in active voice:

(6) Template: $N P_{0} V G$

$$
\begin{aligned}
& \langle V G \text { head }\rangle=V_{t r} \\
& \langle V G \text { head voice }\rangle=\text { active } \\
& \langle\text { example }\rangle=\text { "Runx3 binds" }
\end{aligned}
$$

(7) Template: $V G N P_{1}$

$$
\begin{aligned}
& \langle V G \text { head }\rangle=V_{t r} \\
& \langle V G \text { head voice }\rangle=\text { active } \\
& \langle\text { example }\rangle=\text { "expresses KBF1" }
\end{aligned}
$$

An example template for a verbal predicate $V_{t r}$ in passive voice is:

(8) Template: $N P_{1} V G$

$$
\begin{aligned}
& \langle V G \text { head }\rangle=V_{t r} \\
& \langle V G \text { head voice }\rangle=\text { passive } \\
& \langle\text { example }\rangle=\text { "OTF-1 is expressed" }
\end{aligned}
$$

We use $\mathrm{NP}_{1}$ in pattern templates (7) and (8) in contrast to $\mathrm{NP}_{0}$ in template (6), because their roles are different. For example, in trigger specification of (1), $\mathrm{NP}_{0}$ is always the agent and $\mathrm{NP}_{1}$ is always the theme. Furthermore, in combination with the constraints expressed within the trigger specification, the use of template (6) will succeed only if $\mathrm{NP}_{0}$ is GGP, whereas the use of template (8) will succeed even if $\mathrm{NP}_{1}$ is a protein part.

Nominal triggers In addition to the standard pattern templates that are based on verbal forms of the trigger, we also consider cases where the trigger verb is nominalized $\left(\mathrm{N}_{\mathrm{tr}}\right)$. For example, "transcribe" can be nominalized into "transcription" or "transcript". Nominalization of verbs can be divided into two classes. The first class is where resulting nouns denote actions, states, and processes. Their suffixes are typically "-ion", "-age", and "-ance" (e.g., "transcribe" $\rightarrow$ "transcription", "cleave" $\rightarrow$ "cleavage", and "appear" $\rightarrow$ "appearance"). The second class is where resulting nouns refer to entities (e.g., "transcribe" $\rightarrow$ "transcript" and "produce" $\rightarrow$ "product"). Because our primary interest is processes pertaining to genes or proteins, we currently focus on the first class. Typical pattern templates for nominal triggers are:

(9) Template: $N P_{t r}$ of $N P_{1}$

$$
\begin{aligned}
& \left\langle N P_{t r} \text { head }\right\rangle=N_{t r} \\
& \langle\text { example }\rangle=\text { "expression of IFN-gamma" }
\end{aligned}
$$

(10) Template: $\left[{ }_{N P} N P_{1} N P_{t r}\right]$

$$
\begin{aligned}
& \left\langle N P_{t r} \text { head }\right\rangle=N_{t r} \\
& \langle\text { example }\rangle=\text { "c-fos expression" }
\end{aligned}
$$

Besides the theme, the agent can be incorporated via a "by" phrase. A pattern template for such instances is:

(11) Template: $N P_{\text {tr }}$ by $N P_{0}$

$$
\begin{aligned}
& \left\langle N P_{t r} \text { head }\right\rangle=N_{t r} \\
& \langle\text { example }\rangle=\text { "phosphorylation by Cdk5" }
\end{aligned}
$$

As for non-directional relations we discussed earlier, we have additional templates, which are exemplified by the following:

(12) Template:

$$
\begin{aligned}
& {\left[{ }_{N P} N P_{t r} \text { of } N P_{0} \text { and } N P_{1}\right] \text { or }} \\
& {\left[N P N P_{t r} \text { between } N P_{1} \text { and } N P_{0}\right]}
\end{aligned}
$$$$
\langle\text { direction }\rangle=\text { non-directional }
$$$$
\left\langle N P_{t r} \text { head }\right\rangle=N_{\text {tr }}
$$$$
\left\langle N P_{0} \text { role }\right\rangle \leftarrow \text { theme }
$$$$
\left\langle N P_{1} \text { role }\right\rangle \leftarrow \text { theme }
$$$$
\langle\text { example }\rangle=\text { "binding of p50 and p65" }
$$

Adjectival triggers English has a general morphological process of adjective conversion (Adjtr), which enables verbs to be used as adjectives. The pattern template for adjective triggers is

(13) Template: $\left[{ }_{N P} A D J N P_{1}\right]$

$$
\begin{aligned}
& \langle A D J \text { head }\rangle=A d j_{t r} \\
& \langle\text { example }\rangle=\text { "expressed pseudogenes" }
\end{aligned}
$$

In this framework, adjectival derivations can be the present participle (14a), the past participle (14b), and the adjectivization (14c) of a verb.

(14) a. its proximal binding element PO

$$
\begin{aligned}
& \text { b. phosphorylated GSK3 } \\
& \text { c. transcriptional protein Sp1 }
\end{aligned}
$$

\section{C.4 Generation of patterns}

The pattern generation module automatically creates lexico-syntactic patterns given a list of trigger specifications and frames.

To associate pattern templates with frames, verb type information is used. For example, one constraint in English is that only transitive verbs can be passivized. Therefore Frame: $N P_{0} / N P_{1}$ contains template (6), (7), and (8), but Frame: $N P_{0}$ contains template (6) only. Given the trigger specification of (1) for "phosphorylate" having Frame: $N P_{0} / N P_{1}$, we will automatically generate lexicosyntactic patterns like " $\mathrm{NP}_{0}$ phosphorylates", "NP${ }_{1}$ is phosphorylated", etc..

The automatic generation procedure is similar to the concept of LTAG. In LTAG, a tree family associates a verb lexeme with a given subcategorization. The subcategorization includes a set of grammatical structures that represent all the possible lexico-syntactic variations 
for that verb lexeme. So grammatical structures can be obtained by combining lexical rules and syntactic transformations. Compared with LTAG, the "frame" in our approach is essential but not exactly a subcategorization in LTAG. The trigger specifications are similar to tree families in LTAG, which associate a trigger lexeme with a given frame. In addition, we also consider verb nominalization and adjectivization.

\section{C.5 Pattern templates with null argument}

There are cases when the writing style does not follow the common trigger-argument association. When the argument is omitted, but implied, we call them "elliptical construction". Following are some examples of (a) elliptical constructions, and (b) how they would be written if the trigger-argument association would be required.

(15) a. When phosphorylated, PI-1 inhibits PP-1.

b. When is phosphorylated, PI-1 inhibits PP-1.

(16) a. LMP1 activated NF-kappa B via phosphorylation.

b. LMP1 activated NF-kappa $B$ via phosphorylation.

Both (a) and (b) are grammatically correct and express the same underlying idea in (15) and (16), but we tend to write (a) rather than (b) as a shorthand. Such null argument structure is similar to the null complement anaphora (deep anaphora) in a modern syntactic theory [42] and the implicit argument in a semantic theory [43]. For the relation extraction task, we observe that the elided argument may be found as its antecedent and determined by another trigger that selects it. Our framework recovers them as part of the relation extraction process, by applying for the null argument pattern templates. It should be noted that such elliptical constructions can appear in various positions of a sentence, e.g., at the beginning (15a) or at the end (16a). These templates always rely on the whole sentence construct, therefore are too cumbersome to express. We designed some pattern templates to match sentences like (15a) and (16a). Whether there exists a more general and clearer way to express these types of pattern templates needs to be further explored.

\section{Pattern matching}

Consider the text fragment where "JNK" and "NFAT4" have already been tagged as gene or gene product.

\section{(17) JNK phosphorylates NFAT4}

This fragment is captured by the generated lexicosyntactic patterns derived from Frame: $N P_{0} / N P_{1}$ and the trigger specification "phosphorylate" in (1). The next step is to extract the actual agent and theme. Specifically, pattern template (7) matches the "phosphorylates" word as a trigger, and "NFAT4" as $\mathrm{NP}_{1}$. The trigger specification (1) checks $\mathrm{NP}_{1}$ 's type, which is GGP, and assigns its role for a theme. Therefore, we get $<$ phosphorylates trigger, NFAT $4_{\text {theme }}>$. Similarly, by using pattern template (6) we can extract $<$ phosphorylates trigger, $\mathrm{JNK}_{\text {agent }}>$.

For pattern matching, we would like to mention two issues. First, as illustrated above, the pattern matching engine must be able to check the types of NPs are consistent with those mentioned in the trigger specifications. For this purpose, any method that assigns types to noun phrases or named entities, such as BioNex [39] or Genia tagger [44] can be employed. In our evaluation, we have used the BioNex tool.

Secondly, in order to match a broader range of phrases, we skip verbal auxiliaries and adjuncts for pattern matching. By auxiliary verbs, we mean verbs used to express tense, aspect, modality, etc. By adjuncts, we mean the optional phrases that do not affect the main meaning of a sentence. First, consider the following examples having auxiliary verbs:

\section{(18) a. has also been demonstrated to express gp70 b. PCC 6803 is able to express}

The above examples belong to pattern templates (7) and (8), respectively. However, none of them can be directly matched because of the complex way in which the predicates are expressed. This construction of consecutive verb groups makes basic pattern matching extremely laborious, because of the many variations they can introduce. In this framework, we would like to avoid constructing complex pattern templates, thereby reducing the burden of system development. We notice that (1) syntactically, such consecutive verb groups form a dependent-auxiliary construction: dependent-auxiliary + main-verb, and (2) semantically, the "agent" and "theme" are always related to the last main-verb, rather than the auxiliary. Thus, we match the consecutive verb group as a whole, then choose the last verb as the head of the whole sequence.

Second, let us look at adjuncts in the following sentences:

$$
\begin{aligned}
& \text { a. } \mathrm{p} 45 \text { mRNA is abundantly transcribed in } \\
& \text { the granulocyte fraction of human peripheral } \\
& \text { blood cells. } \\
& \text { b. Abundant expression of erythroid } \\
& \text { transcription factor P45 NF-E } 2 \text { mRNA } \\
& \text { in human peripheral granurocytes. }
\end{aligned}
$$

The most frequent adjuncts that are likely to be skipped are the adverbial adjuncts, e.g. "abundantly" and "in the granulocyte fraction of human peripheral blood cells" in (19a). In addition, adjective-nominal adjuncts are also skipped, e.g "Abundant" and "in human peripheral granurocytes" in (19b). 


\section{E. Sentence simplification}

So far, we have discussed how arguments can be extracted by matching patterns. But even with a large number of patterns automatically generated in the proposed manner, the recall of the resulting system is still low because sentence constructions and writing styles vary considerably in actual text, and the number of variations to be considered is overwhelmingly high. For example, consider sentence (20):

(20) Active $R a f-1$ phosphorylates and activates the mitogen-activated protein (MAP) kinase/extracellular signal-regulated kinase kinase 1 (MEK1), which in turn phosphorylates and activates the MAP kinases/ extracellular signal regulated kinases, ERK1 and ERK2. (PMID 8557975)

It is difficult to extract phosphorylation relation

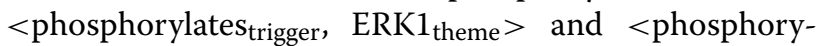
lates trigger, ERK $2_{\text {theme }}>$ from (20) by preconceiving complex patterns required and exhaustively encoding them along with all possible variations. On the other hand, if we can simplify the syntactic structure of (20) and obtain the following sentences, the automatically generated patterns can easily match the simple sentences:

\section{(21) a. Active Raf-1 phosphorylates $M E K 1$. b. MEK1 in turn phosphorylates $E R K 1$. c. MEK1 in turn phosphorylates $E R K 2$.}

This and many other instances that we observed in biomedical research articles motivated us to separate the various structures of a sentence first, and then match patterns to the simplified sentences.

Complex constructs, e.g., coordinations and relative clauses, pose a challenge for state-of-the-art full parsers. However, even if these constructs can be detected correctly using full parsers, new patterns are still needed to skip parts of a construct (e.g., skipping conjuncts in a coordination or skipping relative clauses). When using a dependency parser, more collapsed rules involving prepositions, conjuncts, as well as information about the referent of relative clauses are used to get direct dependencies between content words [45]. Both cases will increase the complexity of patterns and, thus, increase the pattern encoding effort.

Alternatively in this framework, we introduce sentence simplification as a preprocessing module. Given an input sentence, this module outputs a set of generated simplified sentences, thus conceals the syntactic complexity from the pattern matching step.

\section{E.1 Complex constructs for simplification}

In this section, we will describe syntactic constructs that the preprocessing module simplifies. For further details of our sentence simplifier, iSimp, we refer to [33].

Coordinations are syntactic structures that link two or more items (conjuncts) of syntactically equal status [46]. These conjuncts are linked by coordinating conjunctions (e.g., "and", "or", and "but"). Our primary concerns are coordinations of nouns (22a), noun phrases (22b), verbs (22c), and verb phrases (22d).
a. [DNMT] or [MBP]
b. both [the cytoplasm] and [the nucleus]
c. [activates] and [phosphorylates]
d. Foxp3 could [down-regulate ...] and [inhibit ... ]

For a coordination, the original sentence can be split into multiple ones, each containing one conjunct.

Relative clauses are clauses that modify noun phrases. For example,

(23) a. a complex tertiary structure [that is essential for their function]

b. the percentage of cells [expressing IFN-gamma]

c....that is dependent on the interaction of $\mathrm{CD} 40$ with CD40 ligand [expressed on activated T cells]

There are two types of relative clauses that frequently appear in biomedical text: full relative clauses and reduced relative clauses. Full relative clauses (23a) are introduced by relative pronouns, such as "which", "who", and "that". Reduced relative clauses (23b) and (23c) start with a gerund or past participle and have no overt subject. A sentence containing a relative clause can be simplified into two sentences: the original sentence without the relative clause and the other that consists of referred noun phrase as a subject and the relative clause.

Appositions are constructs of two noun phrases next to each other, typically separated by comma and referring to the same entity. For example,

\section{(24) a. the lymphocyte-specific protein tyrosine kinase, [p56lck] \\ b. BV-173, [a bcr-abi-positive B-cell line]}

Appositions can be detected by searching for two noun phrases separated by a comma, when they are not part of a coordination. In addition, because one noun phrase (appositive) normally renames or describes the other, it usually begins with a determiner or a number (as shown above). Appositions can be simplified into two sentences: one with the referred noun phrase and the other with the appositive. 
Parenthesized elements are any words enclosed within "()", "[]", and " \{\} ". They usually refer to or describe preceding noun phrases.

$$
\text { (25) a. chronic myeloid leukemia (CML) }
$$$$
\text { b. AzadC (or AzaC) }
$$

When simplifying parenthesized elements, an additional sentence is created only with the parenthesized elements without the preceding nouns phrase.

\section{E.2 Dealing with attachment ambiguities}

Attachment of phrases poses one of the well-known problems in syntactic ambiguity.

a. a dominant negative mutant of [referred NP TRAF2] that is involved in TNF signaling

b. [referred NP a specific property] of immortalized cells that can not be explained only by ...

a. [NP coordination UV irradiation and H202 treatment] of T lymphocytes

b. methylational status and [NP-PP mRNA expression of DNA methyltransferases]
a. [monocyte adhesion] and [IFN-gamma]

b. PCR [primers and conditions]

Examples in (26) refer to relative clause attachment ambiguities, where there is a complex NP of the type "NP 1 prep $\mathrm{NP}_{2}$ " followed by a relative clause. In such cases, it is unclear whether to attach the relative clause to the first noun phrase $\left(\mathrm{NP}_{1}\right)$ or the second one $\left(\mathrm{NP}_{2}\right)$. Other kinds of attachment ambiguity include PP-attachment, e.g., "NP 1 and $\mathrm{NP}_{2} \mathrm{PP}$ " (27), and the attachment involving coordination, e.g., "Adj $\mathrm{NP}_{1}$ and $\mathrm{NP}_{2}$ " (28). Solving the attachment problem is important in sentence simplification, but we believe it is not a purely syntactic problem [47]. Semantic information is also necessary to make a decision. Therefore, in this study, we produce alternative attachments as candidates while simplifying sentences, and leave the decision to the pattern matching module where type information is available.

\section{F. Referential relation linking}

By using patterns and sentence simplification, the system can detect textual expressions in the argument position. Sometimes, the referred entity is mentioned somewhere else in the text. Consider example (29). The system can extract binding relation $<$ dimerized $_{\text {trigger }}$, the protein $_{\text {theme }}>$ from (29), but the actual target entity is "c-Fox". To link these phrases, we developed patterns to extract referential relations.

(29) The stability of c-Fox was decreased when the protein was dimerized with phosphorylated c-Jun.

\section{F.1 Referential relations}

Referential relation patterns are designed to extract the relationship of one nominal phrase to another, when one provides the necessary information to interpret the other [48]. By utilizing referential relations, an extraction system is able to identify an actual target entity beyond the initially extracted arguments.

Co-referential relations (or co-references) occur when multiple expressions refer to the same referent. For instance, in the previous example, "the protein" and "c-Fox" both refer to the same object. In a co-referential relation, the anaphoric reference can be a pronoun or definite noun phrase, and its antecedent can be the actual name of protein or gene. In this study, co-referential relations are not extracted, except for the case of a relative pronoun, because we consider their detection as a separate and independent task from pattern-based extraction.

Part-whole relations are useful when an argument extracted for a trigger comprises a part of the target entity. For example:

(30) Both Eomes and Runx3 bind at the Prf1 locus.

For biomedical information extraction, this framework focuses on relations between protein parts and a protein, e.g., a residue in a protein. Such part-whole relations in example (30) can be captured by patterns like "NP ${ }_{\text {whole }}$ contains $\mathrm{NP}_{\text {part }}$ " or by the existence of keywords like "locus", "promoter", and "domain".

Member-collection relations are useful in linking a generic reference to a group of entities that are specified in other places in text. For example:

(31) expression of adhesion molecules including integrin alpha, L-selectin, ICAM-3, and H-CAM

The above example illustrates that the generic reference "adhesion molecules" can be extracted as an argument of the trigger "expression". Meanwhile, specific referred entities include "integrin alpha", "L-selectin", "ICAM-3" and "H-CAM". We consider patterns like "NP, such as NP $(, \mathrm{NP})$ *" to identify this type of relations.

Hyponymy relations are used when argument $X$ is a hyponym of argument $\mathrm{Y}$, if $\mathrm{X}$ is a subtype of $\mathrm{Y}$, or when an instance of $\mathrm{X}$ refers to a concept $\mathrm{Y}$. Thus, in (32a), "CD14" is said to be a hyponym of "membrane glycoprotein", and in (32b), "p130 Crk-associated substrate (Cas)" is a hyponym of "protein". When linked, the system extracts <expressed trigger, $\mathrm{CD} 14_{\text {theme }}>$ and $<$ phosphorylated $_{\text {trigger }}, \mathrm{Cas}_{\text {theme }}>$, respectively.

\section{a. CD14 is a membrane glycoprotein expressed specifically on ...}

b. p130 Crk-associated substrate (Cas) was originally identified as a highly phosphorylated protein. 
To achieve this goal, we identify the fragments having keywords such as "acts as" or "is identified as", which are similar to the ones in [49] and [50]. Moreover, the apposition construct can also hold a hyponymy relation between the appositive and the referred noun phrase.

\section{F.2 Linking entities through referential relations}

We will use the example in Figure 2 to illustrate integrating basic patterns and linking relations.

This example contains one transcription relation. Our goal is to extract its trigger and argument, namely $<$ transcribed $_{\text {trigger }}$, tumor necrosis factor alphatheme $>$ which are highlighted in the sentence. We assume "tumor necrosis factor alpha" is typed as a gene.

Given the trigger "transcribe" and using pattern template (8) as discussed earlier, we can extract <transcribed $_{\text {trigger }}$, the earliest genes theme $>$. But "the earliest genes" is not a named entity (This can be discovered by using a named entity recognition tool). In addition, we extract one member-collection relation $<$ one $_{\text {member }}$, the earliest genes collection $>$ and one hyponymy relation $<$ tumor necrosis factor alphahyponym, one hypernym $>$. The first relation enables us to infer $<$ transcribed trigger $_{\text {, }}$ one $_{\text {theme }}>$, since the collection of genes ("the earliest genes") are "transcribed" and, then, one of its members can be "transcribed" as well. Then, the latter relation allows us to state "tumor necrosis factor alpha" is the "one" in this context and hence to conclude $<$ transcribed trigger $_{\text {, }}$ tumor necrosis factor alphatheme $>$.

The algorithm for the linking is as follows. First we collect all referential relations in the document. Then we use the patterns to get instances for a trigger. If the instance's argument is not an informative reference, we recursively search for all of its references in the detected referential relations. If an appropriate reference of an entity is found, we link it to the trigger, by creating a new pair < trigger, referred entity $>$. This search procedure ends when we exhaust all possibilities. As a result, more than one pair may be created and all pairs are proposed.

\section{G. Evaluation design}

Our framework is designed to extract a variety of relations. For the evaluation of our framework, we need test sets containing different types of relations. Furthermore, the data set should include trigger annotations needed to automatically generate patterns. We chose to use the corpora of BioNLP-ST (Shared Tasks) 2011 and 2013 GE tasks, which included several event extraction subtasks [51,52].

\section{G.1 BioNLP-ST GE task}

The BioNLP-ST GE task series aim to extract various events from biomedical text. The first shared task workshop was held in 2009, and the most recent one in 2013. In this study, both 2011 and 2013 corpora are used for the evaluation. We will refer to them as "GE 2011" and "GE 2013" hereafter.

In GE 2011 task, evaluation results were reported on (W)hole, (A)bstract, and (F)ull paper collections, respectively. The abstract collection contains paper abstracts, the full text collection contains full papers, and the whole collection contains both abstracts and full text. Following the same setting, we also report our results on W, A, and F. GE 2011 corpus covers nine types of events: Gene_expression, Transcription, Localization, Protein_catabolism, Phosphorylation, Binding, Regulation, Positive_regulation, and Negative_regulation. Among these, we focused on events with simple entities as themes. Thus, Regulation and its subtypes were removed because their themes could be other events with other triggers. As a result, only the first 6 types of events were evaluated. The first five events were called "Simple Event" collectively. In the GE 2013 corpora, we consider the same events as well.

\section{G.2 Trigger selection}

Since our approach requires a list of triggers, we used the triggers annotated in the corpus. To effectively evaluate our framework, we further decided to focus on a selected group of triggers. Among triggers in GE corpus, we chose only the triggers that are based on verbs (e.g., phosphorylate) and their nominal and adjective forms (Table 1) as discussed before. We did not use the triggers that are pure nouns (e.g., level) or adjectives (e.g., positive). Additionally, we eliminated verb triggers like "find" and "form" because they are not specific to particular biomedical events.

\section{G.3 Evaluation measurement}

The evaluation was carried out by comparing the predicted annotation with the gold standard. We used the

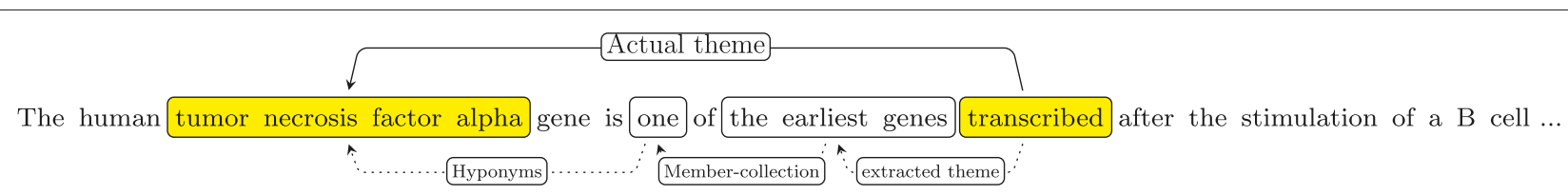

Figure 2 An example of the referential relation linking. The pattern will extract "the earliest genes" as the theme first. Then with the member-collection and hyponymy relations linking, the framework can identify "tumor necrosis factor alpha" as the actual theme of "transcribed". 
Table 1 Selected triggers

\begin{tabular}{|c|c|c|}
\hline Events & Verb & Derivation \\
\hline \multirow[t]{2}{*}{ Gene_expression } & express & -ion, over-, co-, non-, re- \\
\hline & produce & -ion, non-, co- \\
\hline \multirow[t]{4}{*}{ Transcription } & express $^{1}$ & See above \\
\hline & initiate & -tion \\
\hline & produce $^{1}$ & See above \\
\hline & transcribe & -tion, -tional, -tionally \\
\hline \multirow[t]{3}{*}{ Protein_catabolism } & cleave & -age \\
\hline & degrade & -tion, -tive \\
\hline & proteolyse & -sis, -tic, -tically \\
\hline Phosphorylation & phosphorylate & -ion, under-, hyper- \\
\hline \multirow[t]{13}{*}{ Localization } & accumulate & -ation \\
\hline & appear & -ance \\
\hline & detect & \\
\hline & export & \\
\hline & express $^{2}$ & See above \\
\hline & import & \\
\hline & localize & -ation, co-, re- \\
\hline & locate & -ion, re-, trans- \\
\hline & migrate & $\mathrm{CO}^{-}$ \\
\hline & mobilize & -ation, im- \\
\hline & release & \\
\hline & secrete & -ion \\
\hline & transport & \\
\hline \multirow[t]{9}{*}{ Binding } & associate & -ion \\
\hline & bind & DNA- \\
\hline & engage & -ment \\
\hline & interact & -ion \\
\hline & ligate & -ion, Co- \\
\hline & link & cross- \\
\hline & oligomerize & -ation \\
\hline & recruit & -ment \\
\hline & immunoprecipitate & $\mathrm{CO}^{-}$ \\
\hline
\end{tabular}

1. This predication is always used together with "mRNA".

2. This predication is always used together with "surface".

The Derivation column shows affix used to derive other forms of triggers.

Singular, past tense, and gerund forms are not shown.

approximate recursive matching decomposition mode as in the GE task [51], which requires extracting equality between the two events as follows:

1. the event types are the same;

2. the triggers are the same; and

3 . the arguments are the same.

Same triggers and arguments means that "the given text span is equivalent to a gold span if it is entirely contained within an extension of the gold span by one word both to the left and to the right." For example, if $\left(a_{1}, b_{1}\right)$ is the given span and $\left(a_{2}, b_{2}\right)$ is the gold span, they are the same iff $a_{1} \geq a_{2}$ and $b_{1} \leq b_{2}$.

\section{G.4 System implementation}

This section describes one implementation of the framework.

The raw text was parsed by Charniak-Johnson parser using David McClosky's biomedical model [53]. We chose Charniak-Johnson parser because it was convenient in comparing the evaluation with existing systems $[54,55]$. But other constituent parsers would also work with little integration effort.

We consider the typing as a critical component of the framework. For example, (1) for relations like phosphorylation, the theme needs to be a noun phrase of type protein or protein part; (2) for triggers like "associate", the binding relation should not be extracted if its themes' are not proteins or protein parts; and (3) for triggers like "express" and "detect", the themes' type must be gene or mRNA, and the relation is either gene expression or transcription, respectively. This implementation of the framework uses a modified version of BioNex, which was developed based on ideas from [39] and used in RLIMS-P [12]. BioNex can detect semantic types of entities referred by nouns or noun phrases, such as protein, gene, chemical or their part. The type detection is based on considering the head nouns and their suffixes, and comparing them with a predefined list for each type.

Patterns were generated and matched from the parse tree using the tree regular expression [56]. Thus pattern templates were designed using tree regular expression as well. 26 pattern templates were created. To extract the predicate in a consecutive verb group, (e.g., "bind" in "is known to bind"), we looked at the verb phrase subtree and searched for its rightmost children. When the last verb phrase in the group was found, we picked its head.

For the simplification task, we used iSimp, which is a sentence simplifier specifically created for biomedical text [33]. Currently, iSimp can detect six types of simplification constructs: coordination, relative clause, apposition, introductory phrase, subordinate clause, and parenthetical element. It uses shallow parsing and state transition networks to detect all forms of simplifications. The detection of various simplification constructs is based on the chunks (noun phrases, verb groups, and prepositional phrases), and from these, iSimp generates simplified sentences. iSimp also handles nested constructs. For an indepth description of this process, we refer the reader to [33].

For anaphora resolution, we used JavaRAP, which is based on the algorithm of [57] and implemented by [58]. Other referential relation patterns were defined using tree regular expressions. 
The discussion above describes an implementation of the framework. In order to evaluate the framework using the BioNLP-ST GE data, we implemented a relation extraction system for the six events in these data sets. The relation extraction system is obtained from this implementation by specifying the triggers, which were chosen by considering a subset of the trigger words marked in the training set for the six events in the GE 2011 training set. In particular, we chose only frequently occurring verbal trigger words. Note the trigger specifications require only the base form of these verbal triggers (e.g., "phosphorylate" and "interact"). Because this set of triggers are limited in the subcategorization variety, they fall into a handful set of predefined trigger specifications. As a result, we are able to quickly complete the trigger specification for these words.

This relation extraction system implementation is available as a web service accessible: http://research. bioinformatics.udel.edu/ixtractr. Unlike the evaluations conducted in this paper, the web service does not have gene mentions marked in the text as the input. Instead, we integrated an in-house module to detect gene mentions. Because this module only accepts PMIDs as the input rather than full text, the current web service only supports PMID input as well.

\section{Results and discussion}

\section{A. Results on GE 2011 corpus}

After trigger selection, events related to the selected triggers were found to be very frequent in the corpus, covering $81.46 \%$ and $78.78 \%$ of all events in the training and development sets of the GE 2011 corpus, respectively (Table 2). This intrinsic limitation, however, led to an upper bound of $89.78 \%$ and $88.13 \%$ in the F-score of our system.

Table 3 summarizes the performance of our system on the training set of the GE 2011 corpus. We provide

Table 2 Statistics of the data sets after modification

\begin{tabular}{lrrrr}
\hline Events & \multicolumn{2}{r}{$\begin{array}{r}\text { Training set } \\
\text { (\%) }\end{array}$} & \multicolumn{2}{c}{ Development set } \\
& & (\%) \\
\hline Simple Event & 3,165 & 84.92 & 923 & 80.19 \\
$\quad$ Gene_expression & 2,094 & 86.64 & 614 & 79.23 \\
$\quad$ Transcription & 511 & 72.59 & 115 & 69.28 \\
$\quad$ Protein_catabolism & 105 & 92.11 & 22 & 95.65 \\
$\quad$ Phosphorylation & 185 & 94.87 & 107 & 95.54 \\
$\quad$ Localization & 270 & 90.91 & 65 & 86.67 \\
Binding & 874 & 71.00 & 380 & 75.55 \\
Total & 4,039 & 81.46 & 1,303 & 78.78
\end{tabular}

Statistics of events with selected triggers on BioNLP-ST 2011 ST GE task. If an event's argument is within an equivalence relation with $n$ members, this event will be counted $n$ times. $\%=$ Events with selected triggers/All events. results for the Simple Event averaging over five events, results for each of the six individual events including Binding, as well as the overall results for all events. Overall, we obtained a global F-score of $77.78 \%$ for the Simple Event and $65.14 \%$ for the Binding Event. The second part of the results shows the Precision/Recall/F-score when we limited the task to subset events containing only selected triggers. Here, we achieved an F-score of $85.18 \%$ for the Simple Event and $79.44 \%$ for the Binding Event. We also noted that our system attain a higher precision $(>90 \%)$ and a higher recall $(>70 \%)$ on this data subset.

Table 4 summarizes our results on the development set of the GE 2011 corpus. On the whole set, F-scores are reported as $75.10 \%$ for the Simple Event and $62.20 \%$ for the Binding Event. On the subset, F-scores are $84.27 \%$ and $73.55 \%$, respectively. In our experiment, the development set of GE 2011 task contains 1,654 events while the training set contains 4,956 events. Therefore, the numbers on development sets are somewhat lower than those on the training set because a single error or missing case has a higher impact on the overall performance on this smaller data set.

Table 5 shows the effects of different system components on the overall results of our system. We considered three scenarios: (1) using only the argument and null argument patterns; (2) using also the sentence simplification; and (3) using both sentence simplification and referential relation linking. Note that the result of scenario (3) is the same as in the second part of Table 3. Overall, sentence simplification increased the recall by $23 \%$, while referential relation linking achieved an additional $8 \%$ increase. Likewise, results for the development set shows an increase of $22 \%$ and an additional $7 \%$ in recall by simplification and referential relation, respectively (Table 6). Results in both tables indicated that without increasing the number of patterns, simplification and referential relation linking are helpful in extracting more instances of relations.

Table 7 shows results from the test set of 2011 GE tasks. Our system achieves an overall F-score of $72.66 \%$ for the Simple Event, as compared to the F-score of $73.90 \%$ which was the best score for the Simple Event subtask on the GE 2011 test set [51]. Our system achieves an F-score of $55.57 \%$ for the Binding Event, as compared to the F-score of $48.79 \%$ which was the best score for the Binding Event subtask on the GE 2011 test set. The best rule-based system achieved F-scores of $70.52 \%$ and $36.88 \%$ with Simple and Binding events, respectively. Thus, our results compare favorably with those of the top-achieving systems that participated in the GE 2011 task.

We would like to note that although Table 3, 4, 5, 6, and 7 show the results on different partitions of the 2011 data sets, the system remains unchanged because the trigger 
Table 3 Evaluation results on the whole, abstract, and full paper collections from the training set of BioNLP-ST 2011 GE task

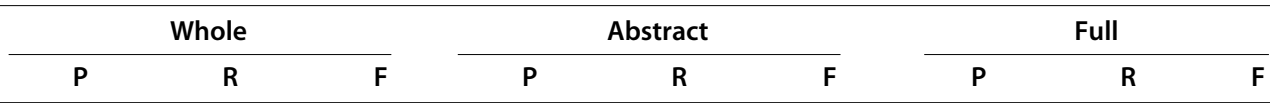

\section{Whole set}

\begin{tabular}{|c|c|c|c|c|c|c|c|c|c|}
\hline Simple event & 92.40 & 67.16 & 77.78 & 93.11 & 66.87 & 77.84 & 89.36 & 68.44 & 77.52 \\
\hline Gene_expression & 92.27 & 69.18 & 79.07 & 93.01 & 68.82 & 79.11 & 89.86 & 70.43 & 78.96 \\
\hline Transcription & 92.43 & 55.54 & 69.39 & 92.88 & 55.67 & 69.61 & 89.66 & 54.74 & 67.97 \\
\hline Protein_catabolism & 91.01 & 71.05 & 79.80 & 93.10 & 71.05 & 80.60 & & - & \\
\hline Phosphorylation & 97.42 & 77.44 & 86.29 & 97.78 & 76.74 & 85.99 & 95.00 & 82.61 & 88.37 \\
\hline Localization & 90.43 & 70.03 & 78.94 & 91.24 & 70.46 & 79.52 & 76.92 & 62.50 & 68.97 \\
\hline Binding & 90.83 & 50.77 & 65.14 & 90.76 & 50.68 & 65.04 & 91.43 & 51.61 & 65.98 \\
\hline Total & 92.08 & 63.10 & 74.88 & 92.60 & 62.57 & 74.68 & 89.61 & 65.83 & 75.90 \\
\hline \multicolumn{10}{|c|}{ Subset with selected triggers } \\
\hline Simple event & 92.29 & 79.08 & 85.18 & 92.98 & 78.92 & 85.38 & 89.36 & 79.79 & 84.31 \\
\hline Gene_expression & 92.27 & 79.85 & 85.61 & 93.01 & 79.25 & 85.58 & 89.86 & 81.94 & 85.71 \\
\hline Transcription & 92.00 & 76.52 & 83.55 & 92.37 & 77.93 & 84.54 & 89.66 & 68.42 & 77.61 \\
\hline Protein_catabolism & 91.01 & 77.14 & 83.51 & 93.10 & 77.14 & 84.38 & & - & \\
\hline Phosphorylation & 97.42 & 81.62 & 88.82 & 97.78 & 81.48 & 88.89 & 95.00 & 82.61 & 88.37 \\
\hline Localization & 90.04 & 77.04 & 83.03 & 90.83 & 77.65 & 83.72 & 76.92 & 66.67 & 71.43 \\
\hline Binding & 90.25 & 70.94 & 79.44 & 90.11 & 70.47 & 79.09 & 91.43 & 75.29 & 82.58 \\
\hline Total & 91.88 & 77.32 & 83.97 & 92.35 & 76.95 & 83.95 & 89.61 & 79.22 & 84.09 \\
\hline
\end{tabular}

Performance is reported in terms of (P)recision/(R)ecall/(F)-score.

Table 4 Evaluation results on the whole, abstract, and full paper collections from the development set of BioNLP-ST 2011 GE task

\begin{tabular}{|c|c|c|c|c|c|c|c|c|c|}
\hline & \multicolumn{3}{|c|}{ Whole } & \multicolumn{3}{|c|}{ Abstract } & \multicolumn{3}{|c|}{ Full } \\
\hline & $\mathbf{P}$ & $\mathbf{R}$ & $\mathrm{F}$ & $\mathbf{P}$ & $\mathbf{R}$ & $F$ & $\mathbf{P}$ & $\mathbf{R}$ & $\mathrm{F}$ \\
\hline \multicolumn{10}{|l|}{ Whole set } \\
\hline Simple event & 92.06 & 63.42 & 75.10 & 92.04 & 65.61 & 76.61 & 92.08 & 61.05 & 73.42 \\
\hline Gene_expression & 92.28 & 64.77 & 76.12 & 91.01 & 66.75 & 77.02 & 93.61 & 62.88 & 75.23 \\
\hline Transcription & 89.13 & 49.40 & 63.57 & 94.55 & 57.78 & 71.72 & 81.08 & 39.47 & 53.10 \\
\hline Protein_catabolism & 94.12 & 69.57 & 80.00 & 93.75 & 71.43 & 81.08 & 100.00 & 50.00 & 66.67 \\
\hline Phosphorylation & 98.77 & 71.43 & 82.90 & 96.77 & 62.50 & 75.95 & 100.00 & 78.13 & 87.72 \\
\hline Localization & 84.75 & 66.67 & 74.63 & 91.49 & 70.49 & 79.63 & 58.33 & 50.00 & 53.85 \\
\hline Binding & 91.51 & 47.12 & 62.20 & 86.96 & 42.94 & 57.49 & 98.98 & 54.80 & 70.55 \\
\hline Total & 91.92 & 58.46 & 71.47 & 90.65 & 57.62 & 70.46 & 93.53 & 59.53 & 72.76 \\
\hline \multicolumn{10}{|l|}{ Subset with selected triggers } \\
\hline Simple event & 91.17 & 78.33 & 84.27 & 91.10 & 78.74 & 84.47 & 91.26 & 77.86 & 84.03 \\
\hline Gene_expression & 91.18 & 80.78 & 85.66 & 89.93 & 82.51 & 86.06 & 92.48 & 79.10 & 85.27 \\
\hline Transcription & 89.13 & 71.30 & 79.23 & 94.55 & 73.24 & 82.54 & 81.08 & 68.18 & 74.07 \\
\hline Protein_catabolism & 94.12 & 72.73 & 82.05 & 93.75 & 71.43 & 81.08 & 100.00 & 100.00 & 100.00 \\
\hline Phosphorylation & 98.77 & 74.77 & 85.11 & 96.77 & 65.22 & 77.92 & 100.00 & 81.97 & 90.09 \\
\hline Localization & 83.05 & 75.38 & 79.03 & 89.36 & 79.25 & 84.00 & 58.33 & 58.33 & 58.33 \\
\hline Binding & 90.73 & 61.84 & 73.55 & 85.71 & 57.02 & 68.49 & 98.98 & 70.29 & 82.20 \\
\hline Total & 91.06 & 73.52 & 81.36 & 89.63 & 71.60 & 79.61 & 92.89 & 76.01 & 83.61 \\
\hline
\end{tabular}

Performance is reported in terms of (P)recision/(R)ecall/(F)-score. 
Table 5 Comparative results of subset events with selected triggers on the whole, abstract, and full paper collections from the training set of BioNLP-ST $2011 \mathrm{GE}$ task

\begin{tabular}{|c|c|c|c|c|c|c|c|c|c|}
\hline & \multicolumn{3}{|c|}{ Whole } & \multicolumn{3}{|c|}{ Abstract } & \multicolumn{3}{|c|}{ Full } \\
\hline & $\mathbf{P}$ & $\mathbf{R}$ & $\mathrm{F}$ & $\mathbf{P}$ & $\mathbf{R}$ & $\mathrm{F}$ & $\mathbf{P}$ & $\mathbf{R}$ & $F$ \\
\hline \multicolumn{10}{|l|}{ Basic patterns } \\
\hline Simple event & 93.37 & 50.74 & 65.75 & 93.68 & 49.30 & 64.61 & 92.20 & 57.17 & 70.58 \\
\hline Binding & 94.87 & 29.63 & 45.16 & 94.54 & 28.52 & 43.82 & 97.14 & 40.00 & 56.67 \\
\hline Total & 93.58 & 46.17 & 61.84 & 93.81 & 44.44 & 60.31 & 92.64 & 54.97 & 69.00 \\
\hline \multicolumn{10}{|c|}{ Using simplification } \\
\hline Simple event & 93.30 & 73.87 & 82.45 & 94.28 & 73.28 & 82.46 & 89.31 & 76.51 & 82.42 \\
\hline Binding & 92.34 & 51.03 & 65.73 & 92.25 & 49.81 & 64.69 & 92.98 & 62.35 & 74.65 \\
\hline Total & 93.14 & 68.93 & 79.23 & 93.92 & 67.79 & 78.75 & 89.69 & 74.70 & 81.51 \\
\hline \multicolumn{10}{|c|}{ Using simplification and referential relations } \\
\hline Simple event & 92.29 & 79.08 & 85.18 & 92.98 & 78.92 & 85.38 & 89.36 & 79.79 & 84.31 \\
\hline Binding & 90.25 & 70.94 & 79.44 & 90.11 & 70.47 & 79.09 & 91.43 & 75.29 & 82.58 \\
\hline Total & 91.88 & 77.32 & 83.97 & 92.35 & 76.95 & 83.95 & 89.61 & 79.22 & 84.09 \\
\hline
\end{tabular}

Performance is reported in terms of (P)recision/(R)ecall/(F)-score. The third part is reproduced from the second part of Table 3. "Basic patterns" = using pattern templates for argument realization and pattern templates with null argument to generate patterns.

word list (extracted from the training set) remains the same.

\section{B. Analysis of false positives and negatives on GE 2011 corpus}

We randomly chose 50 false positive (FP) cases and 180 false negative (FN) cases with 30 for each event type from the training set of GE 2011 corpora in order to analyze reasons for failure. We identified two major types of errors.

\section{B.1 Parsing errors}

A large proportion of failure was due to errors made by the parser. Since the patterns rely on the parser output, the system failed to recognize a true positive in these cases. Some of the parsing errors were due to noun phrase coordinations. Although the parser detected the coordination, the resulting trees could have been shallow or deep. Figure 3 shows two different parse trees of noun phrase coordinations: (a) is correctly parsed, but (b) is not. Flattening the coordination and applying relaxed matching

Table 6 Comparative results of subset events with selected triggers on the whole, abstract, and full paper collections from the development set of BioNLP-ST 2011 GE task

\begin{tabular}{|c|c|c|c|c|c|c|c|c|c|}
\hline & \multicolumn{3}{|c|}{ Whole } & \multicolumn{3}{|c|}{ Abstract } & \multicolumn{3}{|c|}{ Full } \\
\hline & $\mathbf{P}$ & $\mathbf{R}$ & $\bar{F}$ & $\mathbf{P}$ & $\mathbf{R}$ & $\mathbf{F}$ & $\mathbf{P}$ & $\mathbf{R}$ & $\bar{F}$ \\
\hline \multicolumn{10}{|l|}{ Basic patterns } \\
\hline Simple event & 93.01 & 51.90 & 66.62 & 91.67 & 48.99 & 63.85 & 94.42 & 55.24 & 69.71 \\
\hline Binding & 94.95 & 24.74 & 39.25 & 91.67 & 22.73 & 36.42 & 100.00 & 28.26 & 44.07 \\
\hline Total & 93.32 & 43.98 & 59.78 & 91.67 & 40.35 & 56.04 & 95.17 & 48.68 & 64.41 \\
\hline \multicolumn{10}{|c|}{ Using sentence simplification } \\
\hline Simple event & 92.99 & 74.76 & 82.88 & 92.71 & 74.70 & 82.74 & 93.31 & 74.83 & 83.05 \\
\hline Binding & 94.59 & 46.05 & 61.95 & 91.67 & 45.45 & 60.77 & 100.00 & 47.10 & 64.04 \\
\hline Total & 93.31 & 66.39 & 77.58 & 92.47 & 65.08 & 76.40 & 94.38 & 68.08 & 79.10 \\
\hline \multicolumn{10}{|c|}{ Using sentence simplification and referential relations } \\
\hline Simple event & 91.17 & 78.33 & 84.27 & 91.10 & 78.74 & 84.47 & 91.26 & 77.86 & 84.03 \\
\hline Binding & 90.73 & 61.84 & 73.55 & 85.71 & 57.02 & 68.49 & 98.98 & 70.29 & 82.20 \\
\hline Total & 91.06 & 73.52 & 81.36 & 89.63 & 71.60 & 79.61 & 92.89 & 76.01 & 83.61 \\
\hline
\end{tabular}

Performance is reported in terms of (P)recision/(R)ecall/(F)-score. The third part is reproduced from the second part of Table 4. "Basic patterns" = using pattern templates for argument realization and pattern templates with null argument to generate patterns. 
Table 7 Results on the (W)hole, (A)bstract, and (F)ull paper collections from the testing set of BioNLP-ST 2011 GE task 1

\begin{tabular}{|c|c|c|c|c|c|c|c|c|c|}
\hline \multirow[t]{2}{*}{ Event class } & \multicolumn{3}{|c|}{ Whole } & \multicolumn{3}{|c|}{ Abstract } & \multicolumn{3}{|c|}{ Full } \\
\hline & $P$ & $\mathbf{R}$ & $F$ & $\mathbf{P}$ & $\mathbf{R}$ & $F$ & $\mathbf{P}$ & $\mathbf{R}$ & $\bar{F}$ \\
\hline Simple event & 92.59 & 59.80 & 72.66 & 92.2 & 56.09 & 69.75 & 93.52 & 71.17 & 80.83 \\
\hline Gene_expression & 91.89 & 62.18 & 74.17 & 91.29 & 58.03 & 70.96 & 93.15 & 72.86 & 81.76 \\
\hline Transcription & 93.10 & 46.55 & 62.07 & 92.86 & 47.45 & 62.80 & 94.12 & 43.24 & 59.26 \\
\hline Protein_catabolism & 100.00 & 66.67 & 80.00 & 100.00 & 64.29 & 78.26 & 100.00 & 100.00 & 100.00 \\
\hline Phosphorylation & 92.52 & 73.51 & 81.93 & 91.51 & 71.85 & 80.50 & 95.12 & 78.00 & 85.71 \\
\hline Localization & 96.67 & 45.55 & 61.92 & 97.33 & 41.95 & 58.63 & 93.33 & 82.35 & 87.50 \\
\hline Binding & 89.58 & 40.28 & 55.57 & 89.20 & 35.40 & 50.70 & 90.10 & 51.12 & 65.20 \\
\hline Total & 91.97 & 54.55 & 68.48 & 91.68 & 50.89 & 65.45 & 92.64 & 64.83 & 76.28 \\
\hline
\end{tabular}

Performance is reported in terms of (P)recision/(R)ecall/(F)-score.

rules could have fixed most of these problems. For coordination simplifications in particular, we could apply noun phrase and verb group similarity rules to detect coordination boundary and transform the subtree from (b) to (a) [33].

Parsing errors also cause simplification errors. Figure 4 shows the parsing subtree of the fragment "the physical interaction we detected between Foxp2 and p300". If the parse tree were correct, we could remove the relative clause "we detected" in the simplification step and extract the binding relation between "Foxp3" and "p300", but the incorrect parse tree failed the system. As can be seen,

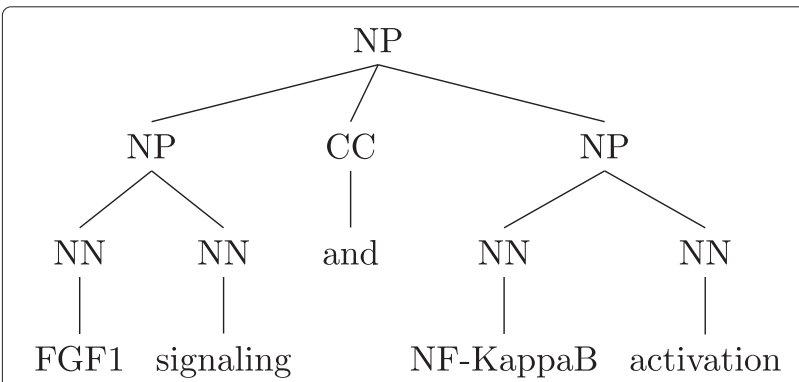

(a)

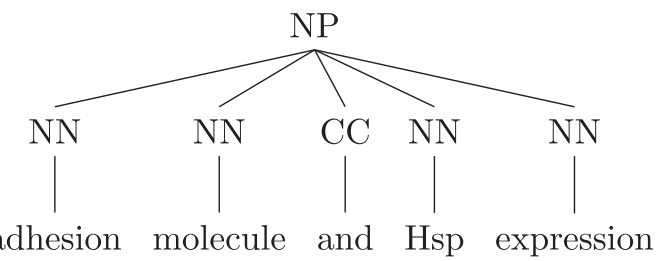

(b)

Figure 3 Two parse trees of coordinations. (a) Parsing tree of the fragment "FGF1 signaling and NF-KappaB activation". (b) Parsing tree of the fragment "adhesion molecule and Hsp expression". errors in sentence simplification can propagate and cause errors in subsequent processing. Most of the simplification errors are due to incorrect coordination detection. However, overall the number of simplification errors are few, and as can be seen from Table 6, the boost in recall is significantly more than the drop in precision.

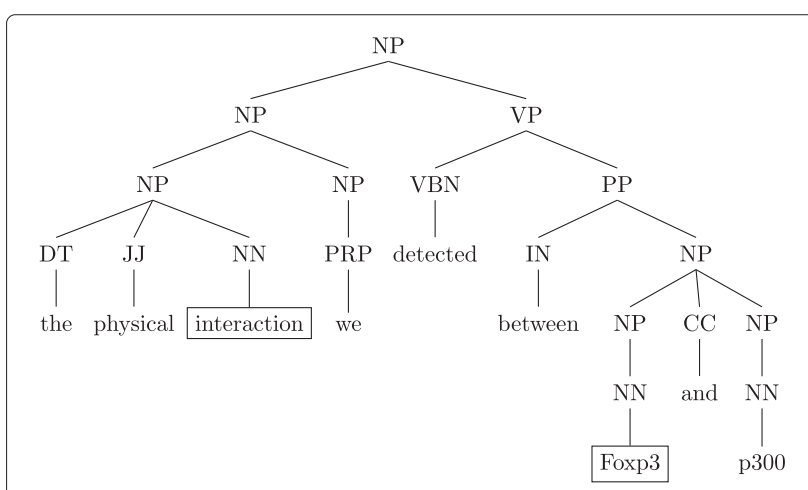

(a): Incorrect parsing tree generated by the parser.

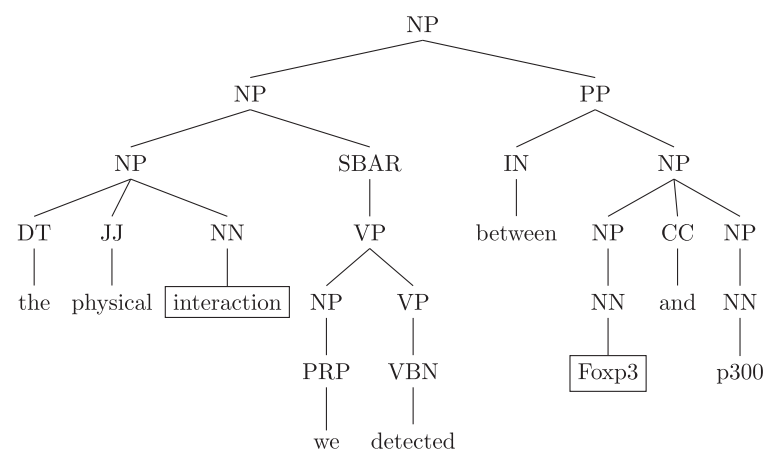

(b): Correct parsing tree.

Figure 4 Two parse trees of the fragment "the physical interaction we detected between Foxp3 and p300". Parse tree of the fragment "the physical interaction we detected between Foxp3 and p300", with target relation <interaction $n_{\text {trigger, }}$ Foxp3 $3_{\text {theme }}>$ highlighted. (a) is the incorrect parse tree generated by the parser. (b) is the correct parse tree. 


\section{B.2 Missing pattern templates}

Another case of false negatives is due to the trigger word being a noun but not the head of the noun phrase. For example, our pattern templates could be applied for fragments "transcription of NP" and "expression of NP" but could not be applied for fragments "[ $\mathrm{NP}_{1}$ transcription rate $]$ of $\mathrm{NP}_{2}$ " or "[ $\mathrm{NP}_{1}$ expression level] of $\mathrm{NP}_{2}$ ". We impose such a constraint in order to maintain a high precision. The analysis showed, however, that we could generalize the constraints in the future with some effort, especially in deciding on the words that can head the NPs.

Similarly, we need to generalize null argument structures further. For example, consider the fragment

\section{(33) targets c-Fos for degradation}

We have a pattern template using "via" but not "for". There are a few other cases, where null argument pattern templates could have been applied, but these new templates need to be further checked.

\section{Results and analysis on GE 2013 corpus}

Table 8 shows the results for the same six events of the GE 2013 test set. We still used the trigger list from the 2011 training set. Thus, the system was the same one used on the 2011 task, without any changes made for the evaluation on the new corpus.

The system achieves F-scores of $80.07 \%$ for the Simple Event and 60.58\% for the Binding Event on the GE 2013 test set with an overall F-scores of $74.44 \%$ on the 2013 GE task. These scores compare favorably with the topranking systems in the $2013 \mathrm{GE}$ task $^{\mathrm{a}}$ [59]. Our system achieves the highest scores for Simple Event and Overall. However there are two participated systems (BioSEM and HDS4NLP), which have better scores for Binding Event. In

Table 8 Evaluation results from the training, development, and testing sets of BioNLP-ST 2013 GE task 1

\begin{tabular}{lccc}
\hline Event class & $\mathbf{P}$ & $\mathbf{R}$ & $\mathbf{F}$ \\
\hline Training set & & & \\
$\quad$ Simple event & 86.75 & 72.20 & 78.81 \\
$\quad$ Binding & 88.95 & 64.83 & 75.00 \\
$\quad$ Total & 87.12 & 70.82 & 78.13 \\
Development set & & & \\
$\quad$ Simple event & 89.52 & 71.73 & 79.64 \\
$\quad$ Binding & 93.58 & 64.42 & 76.31 \\
$\quad$ Total & 90.68 & 69.39 & 78.62 \\
Testing set & & & \\
$\quad$ Simple event & 90.48 & 71.80 & 80.07 \\
$\quad$ Binding & 71.80 & 52.39 & 60.58 \\
$\quad$ Total & 85.27 & 66.05 & 74.44 \\
\hline Performance is reported in terms of (P)recision/(R)ecall/(F)-score. &
\end{tabular}

Performance is reported in terms of (P)recision/(R)ecall/(F)-score. comparison with these systems, our system's strength lies in its precision, achieving $85.52 \%$, whereas, the precision of these systems ranges from $72.90 \%$ to $80.99 \%$.

The testing set of the GE 2013 task is not available to the public, hence we cannot directly examine the results. Instead, we conducted experiments on the training and development sets. Although the results on these two sets are consistent with the corresponding results on 2011 corpora, we noticed some differences between the GE 2013 and 2011 corpora: the former is completely comprised of full-length articles, whereas the latter is mostly made up of abstracts. We also observed that in the full-length articles, certain information is repeatedly mentioned within a single section, therefore there is significant use of ellipses in such sections. For instance, consider the example from the GE 2013 development corpus in Figure 5.

For the trigger "phosphorylation" in the third sentence, the author neglected to mention the theme because it can be inferred from the context: (1) the previous sentence also mentions this "BMP-6 induced phosphorylation", but its theme has a general term "Smad", and (2) the actual proteins "Smad1/5/8" are clearly specified in the first sentence. As a result, to infer the theme of the trigger "phosphorylation" in the third sentence, we not only need the syntax information, but also the discourse-level processing.

Note that the system used in this evaluation remains the same as the one that was used on the GE 2011 task. No changes were made to accommodate any differences between the GE 2011 and 2013 corpora. The focus in this framework is on the patterns and hence almost all processing is syntax-based. While some of our earlier work on relation extraction has integrated discourse-level processing with syntax-based patterns [60], the integration of such discourse-level processing is beyond the scope of this work. However, examples as above suggest that the need for discourse-level processing may be important for full-length based extraction. We intend to investigate incorporating the generalized discourse-level processing into our framework in the future, so that it can be useful for full-text based extraction.

\section{Conclusions}

In this work, we have designed a framework for development of biomedical relation extraction systems. The framework requires as input only a list of triggers and their specifications to retrieve relations of interest. It utilizes linguistic generalizations that help speed up the development process by proposing various lexico-syntactic patterns as well as improve the performance, particularly the recall, by making use of sentence simplification and referential relations.

To evaluate the framework, we developed a relation extraction system, which was produced using general 


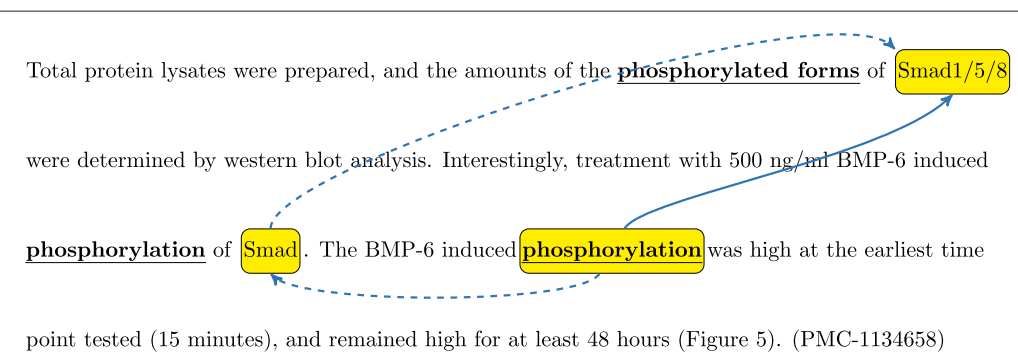

Figure 5 Sample use of ellipses in the paragraph. For the trigger "phosphorylation" in the third sentence, the author neglected to mention the theme because it can be inferred from the context: (1) the previous sentence also mentions this "BMP-6 induced phosphorylation", but its theme has a general term "Smad", and (2) the actual proteins "Smad1/5/8" are clearly specified in the first sentence.

resources and the only aspect specific to the evaluation was the selection of trigger words that appear in the corpus. Except for the specification of triggers, other aspects (parser, typing system, simplification, pattern matching system) are general purpose systems that already existed. The fact that only the specification of the triggers is required from domain experts, together with the fact that no training set is required, meets our goals for developing the framework: ability to create effective relation extraction systems for new relations where resources (e.g., annotated corpus or database) are not publicly available.

We evaluate the performance of the system by producing a relation extraction system and evaluating it on the BioNLP-ST 2011 and 2013 GE tasks. The system achieved F-scores of $68.48 \%$ on the GE 2011 test set, and $74.44 \%$ on the GE 2013 test set. Our analysis shows that we can achieve high precision and good recall with the range of patterns automatically generated from triggers and that simplification and referential relation linking serve to increase the recall while maintaining the precision.

In the future, we would like to extend the framework in two ways. So far, we only considered the triggers that are verbs and their derived forms. Next, we would like to account for triggers that are primarily nouns or adjectives. Also, we would like to extend the framework to take complex entities (e.g., relations themselves) as arguments rather than just simple entities (e.g., genes or proteins).

We are developing systems for additional relations. In general, it is a challenging task to identify all the triggers for the relation and to complete their specifications. This study demonstrates a generalizable relation extraction framework that can be quickly implemented for new relations, initially focusing on a few triggers that appear frequently. While not accounting for a long tail of less frequent triggers, our framework allows additional trigger specifications to be added with little impact on the existing trigger list. Thus as new triggers are found, they can be integrated in the system. Using the framework and this approach, we have developed a system for miRNA-target extraction. Preliminary evaluation based on an in-house corpus of 200 abstracts shows an F-score of the system over $90 \%$ (manuscript in preparation). We would like to use the experience in developing this and other relation extraction to design a process involving user interaction in generating trigger specifications for new relations. In general, the specification of a trigger needs both domain knowledge as well as linguistic knowledge. The domain expert will be able to suggest the trigger words for a relation, whereas linguistic knowledge will be more useful in preparing the trigger specifications of sub-categorization, thematic roles, etc.

In our framework, we already have a predefined set of subcategorization frames and thematic roles that can be utilized in the specifications. This can be used to engage the user in the interactive process. At the beginning, the users who are domain experts will provide a list of trigger words. Then the process will derive various forms of triggers using the linguistic knowledge and ask users to choose. If necessary, the process will use these triggers to generate simple examples for the users to confirm which predefined specification should be associated to the trigger. The whole process will communicate with users in an interactive way, which we expect is able to further speed up the development of new relation extraction systems.

\section{Endnote}

a Simple Event includes Phosphorylation as well, same as in the BioNLP-ST 2011 GE Task 1.

\section{Competing interests}

The authors declare that they have no competing interests.

\section{Authors' contributions}

YP carried out the framework studies described here, conducted the experiments, and drafted the manuscript. MT participated in the design of the study and has been involved in drafting the manuscript. CW coordinated the study, and has been involved in revising the manuscript critically for important intellectual content. KV conceived the study, and participated in its design and coordination and helped in drafting the manuscript. All authors read and approved the final manuscript. 


\section{Acknowledgements}

Research reported in this manuscript was supported by the National Library of Medicine of the National Institutes of Health under award number G08LM010720. The content is solely the responsibility of the authors and does not necessarily represent the official views of the National Institutes of Health. This material is also based upon work supported by the National Science Foundation under Grant No. DBI-1062520. Any opinions, findings, and conclusions or recommendations expressed in this material are those of the author(s) and do not necessarily reflect the views of the National Science Foundation. We thank the BioNLP Shared Task organizer for making the annotated corpora publicly available. We thank Dr. Catalina O. Tudor for useful discussion and comments for this manuscript.

\section{Author details}

${ }^{1}$ Department of Computer and Information Sciences, University of Delaware, 18 Amstel Ave, Newark, DE 19716, USA. ${ }^{2}$ Center for Bioinformatics and Computational Biology, University of Delaware, 15 Innovation Way, Newark, DE 19711, USA.

Received: 9 July 2013 Accepted: 15 August 2014

Published: 23 August 2014

\section{References}

1. Vlachos A, Craven M: Biomedical event extraction from abstracts and full papers using search-based structured prediction. $B M C$ Bioinformatics 2012, 13(Suppl 11):S5

2. Riedel S, McClosky D, Surdeanu M, McCallum A, Manning CD: Model combination for event extraction in BioNLP 2011. In Proceedings of the BioNLP Shared Task 2011 Workshop. Portland, Oregon: Association for Computational Linguistics; 2011:51-55.

3. Björne J, Salakoski T: Generalizing biomedical event extraction. In Proceedings of the BioNLP Shared Task 2011 Workshop. Portland, Oregon: Association for Computational Linguistics; 2011:183-191.

4. Bui QC, Katrenko S, Sloot PM: A hybrid approach to extract protein-protein interactions. Bioinformatics 2011, 27(2):259-265.

5. Kim S, Yoon J, Yang J, Park S: Walk-weighted subsequence kernels for protein-protein interaction extraction. BMC Bioinformatics 2010, 11:107.

6. Miwa M, Sætre R, Miyao Y, Tsujii J: Protein-protein interaction extraction by leveraging multiple kernels and parsers. Int $\mathrm{J}$ Med Inform 2009, 78(12):e39.

7. Airola A, Pyysalo S, Björne J, Pahikkala T, Ginter F, Salakoski T: All-paths graph kernel for protein-protein interaction extraction with evaluation of cross-corpus learning. BMC Bioinformatics 2008 , 9(Suppl 11):S2.

8. Pyysalo S, Airola A, Heimonen J, Björne J, Ginter F, Salakoski T: Comparative analysis of five protein-protein interaction corpora. BMC Bioinformatics 2008, 9(Suppl 3):S6

9. Tudor CO, Vijay-Shanker K: RankPref: Ranking sentences describing relations between biomedical entities with an application. In Proceedings of the 2012 Workshop on Biomedical Natural Language Processing. Montreal, Canada: Association for Computational Linguistic; 2012:163-171.

10. Cohen KB, Verspoor K, Johnson HL, Roeder C, Ogren PV, Baumgartner WA, White $\mathrm{E}$, Tipney $\mathrm{H}$, Hunter L: High-precision biological event extraction: effects of system and of data. Comput Intell 2011, 27(4):681-701.

11. Hakenberg J, Leaman R, Ha Vo N, Jonnalagadda S, Sullivan R, Miller C, Tari $L$, Baral C, Gonzalez G: Efficient extraction of protein-protein interactions from full-text articles. IEEE/ACM Trans Comput Biol Bioinform (TCBB) 2010, 7(3):481-494.

12. Hu ZZ, Narayanaswamy M, Ravikumar KE, Vijay-Shanker K, Wu CH: Literature mining and database annotation of protein phosphorylation using a rule-based system. Bioinformatics 2005 , 21(11):2759-2765.

13. Kilicoglu $H$, Bergler $S$ : Adapting a general semantic interpretation approach to biological event extraction. In Proceedings of the BioNLP Shared Task 2011 Workshop. Portland, Oregon: Association for Computational Linguistics; 2011:173-182.

14. Quirk C, Choudhury P, Gamon M, Vanderwende L: MSR-NLP entry in BioNLP shared task 2011. In Proceedings of the BioNLP Shared Task 2011
Workshop. Portland, Oregon: Association for Computational Linguistics; 2011:155-163.

15. Kim J, Rebholz-Schuhmann D: Improving the extraction of complex regulatory events from scientific text by using ontology-based inference. J Biomed Semantics 2011, 2(Suppl 5):S3.

16. Fundel K, Küffner R, Zimmer R: RelEx - relation extraction using dependency parse trees. Bioinformatics 2007, 23(3):365-371.

17. Rinaldi F, Schneider G, Kaljurand K, Hess M, Andronis C, Konstandi O, Persidis A: Mining of relations between proteins over biomedical scientific literature using a deep-linguistic approach. Artif Intell Med 2007, 39(2):127-136.

18. Hunter L, Lu Z, Firby J, Baumgartner WA, Johnson HL, Ogren PV, Cohen KB: OpenDMAP: an open source, ontology-driven concept analysis engine, with applications to capturing knowledge regarding protein transport, protein interactions and cell-type-specific gene expression. BMC Bioinformatics 2008, 9:78

19. Schabes $Y$ : Stochastic lexicalized tree-adjoining grammars. In Proceedings of the 14th conference on Computational linguistics-Volume 2. Nantes, France: Association for Computational Linguistics; 1992:425-432.

20. Pollard C, Sag IA: Head-driven phrase structure grammar. Chicago: University of Chicago Press; 1994.

21. Bresnan J: Lexical-functional syntax. Hoboken: Wiley-Blackwell; 2001.

22. Kipper K, Korhonen A, Ryant N, Palmer M: Extending VerbNet with novel verb classes. In Proceedings of LREC; Genova, Italy, Volume 2006; 2006:1.

23. Chen J, Vijay-Shanker K: Automated extraction of TAGs from the Penn Treebank. In New Developments in Parsing Technology, Volume 23. New York: Springer; 2005:73-89.

24. The XTAG Research Group: A lexicalized tree adjoining grammar for English. Tech. rep., Technical Report IRCS-01-03, IRCS, University of Pennsylvania 2001.

25. Levin B: English verb classes and alternations: a preliminary investigation. Chicago: University of Chicago Press; 1993.

26. Dolbey AE: BioFrameNet: a FrameNet extension to the domain of molecular miology. PhD thesis. University of California: Berkeley; 2009

27. Rebholz-Schuhmann D, Jimeno-Yepes A, Arregui M, Kirsch H: Measuring prediction capacity of individual verbs for the identification of protein interactions. J Biomed Inform 2010, 43(2):200-207.

28. Lippincott T, Rimell L, Verspoor K, Korhonen A: Approaches to verb subcategorization for biomedicine. J Biomed Inform 2013, 46(2):212-227.

29. Rimell L, Lippincott T, Verspoor K, Johnson HL, Korhonen A: Acquisition and evaluation of verb subcategorization resources for biomedicine. J Biomed Inform 2013, 46(2):228-237.

30. EvidenceFinder. http://labs.europepmc.org/evf.

31. Jonnalagadda S, Gonzalez G: BioSimplify: an open source sentence simplification engine to improve recall in automatic biomedical information extraction. In AMIA Annual Symposium Proceedings; Washington, DC, Volume 2010: American Medical Informatics Association; 2010:351

32. Miwa M, Saetre R, Miyao Y, Tsujii J: Entity-focused sentence simplification for relation extraction. In Proceedings of the 23rd International Conference on Computational Linguistics. Beijing, China; 2010:788-796

33. Peng Y, Tudor CO, Torii M, Wu CH, Vijay-Shanker K: iSimp: A sentence simplification system for biomedical text. In IEEE International Conference on Bioinformatics and Biomedicine (BIBM2012). Philadelphia, PA; 2012:211-216.

34. Ogren PV: Coordination resolution in biomedical texts. PhD thesis. University of Colorado at Boulder; 2011.

35. Jimeno-Yepes A, Jiménez-Ruiz E, Berlanga-Llavori R, Rebholz-Schuhmann $D$ : Reuse of terminological resources for efficient ontological engineering in life sciences. BMC Bioinformatics 2009, 10(Suppl 10):S4.

36. Van Landeghem S, Björne J, Abeel T, De Baets B, Salakoski T, Van de Peer $Y Z$ : Semantically linking molecular entities in literature through entity relationships. BMC Bioinformatics 2012, 13(Suppl 11):S6.

37. Miwa M, Thompson $P$, Ananiadou S: Boosting automatic event extraction from the literature using domain adaptation and coreference resolution. Bioinformatics 2012, 28(13):1759-1765.

38. Van Landeghem S, Pyysalo S, Ohta T, Van de Peer Y: Integration of static relations to enhance event extraction from text. In Proceedings of the 2010 Workshop on Biomedical Natural Language Processing. Uppsala, Sweden: Association for Computational Linguistics; 2010:144-152. 
39. Narayanaswamy M, Ravikumar K, Vijay-Shanker K: A biological named entity recognizer. In Proceedings of the Pacific Symposium on Biocomputing. Kauai, Hawaii; 2003:427.

40. Miller JE, Torii M, Vijay-Shanker K: Building domain-specific taggers without annotated (domain) data. In Proceedings of the 2007 Joint Conference on Empirical Methods in Natural Lanquage Processing and Computational Natural Language Learning. Prague, Czech Republic; 2007:1103-1111.

41. Levin B: Hovar MR: Argument realization. Cambridge, UK: Cambridge University Press; 2005

42. Smith NA: Ellipsis happens, and deletion is how. Univ Md Working Papers Linguist 2001, 11:176-191.

43. Gerber M, Chai JY: Semantic role labeling of implicit arguments for nominal predicates. Comput Linguist 2012, 38(4):755-798.

44. Tsuruoka Y, Tsujii J: Bidirectional inference with the easiest-first strategy for tagging sequence data. In Proceedings of the Conference on Human Language Technology and Empirical Methods in Natural Language Processing 2005. Vancouver, Canada; 2005:467-474.

45. De Marneffe MC, Manning CD: Stanford typed dependencies manual. 2008. [http://nlp.stanford.edu/software/dependenciesmanual.pdf]

46. Huddleston R, Pullum GK: The Cambridge grammar of the English language. Cambridge, UK: Cambridge University Press; 2002.

47. Siddharthan A: Syntactic simplification and text cohesion. University of Cambridge 2003.

48. Hartmann RRK, Stork FC: Dictionary of language and linguistics. New York: Wiley; 1972.

49. Hearst MA: Automatic acquisition of hyponyms from large text corpora. Nantes, France: Association for Computational Linguistics; 1992

50. Snow R, Jurafsky D, Ng AY: Learning syntactic patterns for automatic hypernym discovery. Adv Neural Inform Process Syst 2004, 17:1297-1304.

51. Kim JD, Nguyen N, Wang Y, Tsujii J, Takagi T, Yonezawa A: The genia event and protein coreference tasks of the BioNLP shared task 2011. BMC Bioinformatics 2012, 13(Suppl 11):S1.

52. Kim JD, Yue W, Yamamoto Y: The Genia event extraction shared task, 2013 edition - Overview. In Proceedings of the Workshop on BioNLP Shared Task 2013. Sofia, Bulgaria; 2013:20-27.

53. Stenetorp P, Topić G, Pyysalo S, Ohta T, Kim JD, Tsujii J: BioNLP shared task 2011: Supporting resources. In Proceedings of the Workshop on BioNLP Shared Task 2011. Portland, Oregon; 2011:112-120.

54. McClosky D: Any domain parsing: automatic domain adaptation for natural language parsing. PhD thesis. Department of Computer Science, Brown University 2009.

55. Tateisi Y, Yakushiji A, Ohta T, Tsujii J: Syntax annotation for the GENIA corpus. In Proceedings of the Workshop on the 1st International Joint Conference on Natural Language Processing (IJCNLP). Volume 5. Jeju Island Korea; 2005:222-227.

56. Levy R, Andrew G: Tregex and Tsurgeon: tools for querying and manipulating tree data structures. In Proceedings of the Fifth International Conference on Language Resources and Evaluation. Genoa, Italy: 2006:2231-2234.

57. Lappin S, Leass HJ: An algorithm for pronominal anaphora resolution. Comput Linguist 1994, 20(4):535-561.

58. Qiu $L$, yen Kan M, seng Chua T: A public reference implementation of the rap anaphora resolution algorithm. In Proceedings of the Fourth International Conference on Language Resources and Evaluation (LREC 2004). Lisbon, Portugal; 2004:291-294.

59. BioNLP-ST 2013 GE task results. http://bionlp-st.dbcls.jp/GE/2013/ results.

60. Narayanaswamy M, Ravikumar K, Vijay-Shanker K: Beyond the clause: extraction of phosphorylation information from medline abstracts. Bioinformatics 2005, 21 (suppl 1):i319-i327.

doi:10.1186/1471-2105-15-285

Cite this article as: Peng et al:: A generalizable NLP framework for fast development of pattern-based biomedical relation extraction systems. BMC Bioinformatics 2014 15:285.

Submit your next manuscript to BioMed Central and take full advantage of:

- Convenient online submission

- Thorough peer review

- No space constraints or color figure charges

- Immediate publication on acceptance

- Inclusion in PubMed, CAS, Scopus and Google Scholar

- Research which is freely available for redistribution

Submit your manuscript at www.biomedcentral.com/submit
C Biomed Central 